OPEN ACCESS

Edited by:

Emile Van Den Akker,

Sanquin Research, Netherlands

Reviewed by:

Zhipeng Liu,

Purdue University, United States

Robert F. Paulson,

The Pennsylvania State University

(PSU), United States

*Correspondence:

James J. Bieker

james.bieker@mssm.edu

Specialty section:

This article was submitted to Human and Medical Genomics, a section of the journal

Frontiers in Genetics

Received: 09 August 2021 Accepted: 04 November 2021 Published: 22 November 2021

Citation:

Mukherjee K and Bieker JJ (2021) Transcriptional Control of Gene Expression and the Heterogeneous Cellular Identity of Erythroblastic Island Macrophages.

Front. Genet. 12:756028.

doi: 10.3389/fgene.2021.756028

\section{Transcriptional Control of Gene Expression and the Heterogeneous Cellular Identity of Erythroblastic Island Macrophages}

\author{
Kaustav Mukherjee ${ }^{1,2}$ and James J. Bieker ${ }^{1,2,3,4 *}$ \\ ${ }^{1}$ Department of Cell, Developmental, and Regenerative Biology, Mount Sinai School of Medicine, New York, NY, United States, \\ ${ }^{2}$ Black Family Stem Cell Institute, Mount Sinai School of Medicine, New York, NY, United States, ${ }^{3}$ Tisch Cancer Center, Mount \\ Sinai School of Medicine, New York, NY, United States, ${ }^{4}$ Mindich Child Health and Development Institute, Mount Sinai School of \\ Medicine, New York, NY, United States
}

During definitive erythropoiesis, maturation of erythroid progenitors into enucleated reticulocytes requires the erythroblastic island (EBI) niche comprising a central macrophage attached to differentiating erythroid progenitors. Normally, the macrophage provides a nurturing environment for maturation of erythroid cells. Its critical physiologic importance entails aiding in recovery from anemic insults, such as systemic stress or acquired disease. Considerable interest in characterizing the central macrophage of the island niche led to the identification of putative cell surface markers enriched in island macrophages, enabling isolation and characterization. Recent studies focus on bulk and single cell transcriptomics of the island macrophage during adult steadystate erythropoiesis and embryonic erythropoiesis. They reveal that the island macrophage is a distinct cell type but with widespread cellular heterogeneity, likely suggesting distinct developmental origins and biological function. These studies have also uncovered transcriptional programs that drive gene expression in the island macrophage. Strikingly, the master erythroid regulator EKLF/Klf1 seems to also play a major role in specifying gene expression in island macrophages, including a putative EKLF/Klf1dependent transcription circuit. Our present review and analysis of mouse single cell genetic patterns suggest novel expression characteristics that will enable a clear enrichment of EBI subtypes and resolution of island macrophage heterogeneity. Specifically, the discovery of markers such as Epor, and specific features for EKLF/ Klf1-expressing island macrophages such as Sptb and Add2, or for SpiC-expressing island macrophage such as Timd4, or for Maf/Nr1h3-expressing island macrophage such as Vcam1, opens exciting possibilities for further characterization of these unique macrophage cell types in the context of their critical developmental function.

Keywords: erythroblastic island, mammalian hematopoiesis, macrophage identity, erythropoiesis, transcriptional control, single cell analyses, EKLF/KLF1 


\section{INTRODUCTION}

The erythroblastic island (EBI) niche is one of the earliest observed and described stem cell niches in biology consisting of a central macrophage that is attached to erythroid cells at various stages of differentiation (Bessis, 1958; Mohandas and Prenant, 1978; Bernard, 1991). This macrophage aids erythroid maturation by providing cytokines and growth factors, enables enucleation by providing phagocytic functions, and ultimately provides an effective means for reticulocyte formation and release (reviewed comprehensively in Chasis and Mohandas (2008), Manwani and Bieker (2008), de Back et al. (2014), Hom et al. (2015), Klei et al. (2017), Li et al. (2020)). As a result, considerable effort has been dedicated to characterizing EBI macrophages by studying cell surface marker expression for their efficient isolation (Soni et al., 2006; Porcu et al., 2011; Chow et al., 2013; Seu et al., 2017; Li et al., 2019), with various groups reporting different combinations of markers associated with them (Sadahira et al., 1991; Sadahira et al., 1995; Chow et al., 2013; Seu et al., 2017; Li et al., 2019; Yeo et al., 2019b; Mukherjee et al., 2021; Zhang et al., 2021).

These studies however reveal that EBI macrophages are inherently composed of heterogeneous subpopulations, and distinct subtypes of EBIs have also been reported (Seu et al., 2017; Tay et al., 2020; Zhang et al., 2021). Surprisingly, despite their seemingly crucial role in in vivo erythropoiesis, depletion of EBI macrophages does not affect steady-state erythropoiesis (Korolnek and Hamza, 2015; Ulyanova et al., 2016). However, EBI alterations or defects during steady-state erythropoiesis may not necessarily be manifest as severe phenotypes because adequate coping mechanisms to recover and repair EBIs are only required during stress. This is supported by the fact that EBI macrophages are critical for recovery from stress erythropoiesis in the splenic red pulp (Sadahira et al., 2000; Chow et al., 2013; Ramos et al., 2013; Jacobsen et al., 2015; Liao et al., 2018; May and Forrester, 2020). Recovery is effectively aided by de novo differentiation of monocytes into macrophages (Ulyanova et al., 2016; Liao et al., 2018) and via epo-induced signaling in island macrophage that yields prostaglandin mediators of stress erythroid progenitors (Chen et al., 2020). Clinical reports of altered EBI structures correlating with disease have been observed, particularly after examining the effect of primary myelofibrosis (PMF) on the bone marrow (BM) or of lower than normal macrophage levels in some acute leukemias (Koury, 2014). Modulating the effects of B-thalassemia and polycythemia vera $(\mathrm{PV})$ by targeting the central macrophage have been proposed (Li et al., 2020). Additionally, a significant proportion of myelodysplastic syndrome (MDS) patients have alterations in EBIs, particularly of numerical density and size, that correlate with severe anemia and poor disease prognosis independent of other factors such as age (Buesche et al., 2016).

Until recently, detailed characterizations of EBI macrophage heterogeneity and function were hampered by a lack of global gene expression profiles in these macrophages. This was partly due to uncertainty regarding cell surface markers that would specifically enrich for the subpopulation of macrophages that form the EBI niche (Seu et al., 2017; Tay et al., 2020). F4/80 antigen expression is associated with EBI macrophages (Sadahira et al., 1991; Manwani and Bieker, 2008) although not all F4/80+ macrophages in hematopoietic tissues necessarily form EBIs. Nevertheless, recent studies have used F4/80 in addition to surrogate markers such as pEKLF/GFP or Epor-eGFP to enrich for EBI macrophage subpopulations from primary mouse hematopoietic tissue and have determined global gene expression at the bulk (Xue et al., 2014; Li et al., 2019; Mukherjee et al., 2021) and single cell level (Mukherjee et al., 2021). These studies have uncovered novel aspects of EBI macrophage identity, heterogeneity, and physiological function while reinforcing certain existing paradigms. They have also shed substantial light on some unanswered questions regarding transcriptional control of gene expression in EBI macrophages.

The goal of this review is to summarize the findings of the bulk transcriptomic studies of murine EBI macrophages, and to delve deeper into the single cell RNA-Seq data to reveal new aspects of cellular identity and heterogeneity, and the underlying transcription programs putatively controlling gene expression in EBI macrophages.

\section{THE TRANSCRIPTOMICS OF EPOR+ EBI MACROPHAGES}

Two compelling sets of observations led to the hypothesis that the erythropoietin receptor (Epor) is expressed in EBI macrophages and EPO signaling is important for EBI macrophage function. First, EPO signaling and addition of recombinant human EPO had been noted to increase the expansion and activity of the erythroid and macrophage compartments in mice primary tissue (Lifshitz et al., 2010; Xiang et al., 2015; Luo et al., 2016; Wang et al., 2018). Second, Epor expression is not restricted to erythroid cells and is indeed expressed in wide variety of tissues (discussed in Zhang et al. (2021)). Based on these studies it was hypothesized and demonstrated that BM F4/80+ macrophages express Epor, and that the fraction of F4/80+ macrophages that form EBIs is significantly enriched for Epor+ macrophages, thus establishing a positive correlation between Epor expression in macrophages and propensity for EBI formation (Li et al., 2019). Thus, Epor was established as a novel marker for EBI-enriched macrophages in combination with F4/80 (Paulson, 2019).

This enabled the development of a strategy to use Epor as a marker for isolating EBI macrophages with an Epor-/eGFPcre knock-in mouse. BM F4/80+Epor/eGFP+ and F4/80+Epor/ eGFP- populations (also Ter119-Ly6G-, thus not inclusive of erythroid cells or granulocytes) were separated by FACS and global gene expression in the two sub-populations compared by bulk RNA-Seq (Li et al., 2019). As expected, the two populations show inherently distinct gene expression signatures, with the Epor/eGFP+ macrophages expressing genes specifically involved in EBI niche functions such as iron homeostasis (Hmox1, Slc40a1, Tfrc), cell adhesion (Vcam1, Siglec1), phagocytosis and nuclear engulfment (Mertk, Tim4). In contrast, the Epor/eGFPmacrophages express innate immunity genes involved in functions such as inflammation, macrophage activation, and activation of the adaptive immune response (Li et al., 2019). 
Notably, a few transcription factors including EKLF/Klf1 and Spi$\mathrm{C}$ are enriched in Epor/eGFP+ macrophages, indicating that transcription regulation in EBI macrophages depends on the action of these transcription factors. The liver $\mathrm{X}$ receptor $a$ (Nr1h3), implicated in lipid metabolism in macrophages and inflammation (Zhao et al., 2021), and the transcription factor Maf, which has been shown to regulate F4/80 (Adgre1) and IL-10 expression in macrophages (Cao et al., 2005; Nakamura et al., 2009; Kusakabe et al., 2011), are also enriched in Epor+ macrophages. Elucidating their specific roles in regulating transcription in EBI macrophages is thus a promising avenue for further investigation.

\section{EKLF/KLF1 EXPRESSION AND FUNCTION IN FL F4/80+ MACROPHAGES}

Although EKLF/Klf1 is mostly known for its tissue-restricted, global transcriptional role in facilitating maturation and differentiation of proerythroblasts into mature reticulocytes (Tallack and Perkins, 2010; Siatecka and Bieker, 2011; Gnanapragasam and Bieker, 2017) and in fetal to adult globin switching (Bieker, 2010; Perkins et al., 2016), cellular analyses show that EKLF is also critical for the integrity of EB islands (Xue et al., 2014). Consequently, EBIs isolated from $\mathrm{EKLF}^{-/}$mouse fetal livers (FL) exhibit quite a few aberrations; for example, there are overall fewer F4/80+ macrophages (Mukherjee et al., 2021), and isolated EB islands have severely altered morphology compared to $\mathrm{EKLF}^{+/+}$FL (Xue et al., 2014). Thus, our previous understanding of EKLF's role in erythropoiesis as being restricted to the erythroid compartment required reassessment for which, firstly, EKLF expression in F4/80+ macrophages had to be directly and clearly demonstrated. This was accomplished by purifying F4/80+ macrophages from E14.5 FL and detecting EKLF mRNA expression by RT-PCR and protein expression by immunostaining (Porcu et al., 2011; Mukherjee et al., 2021). Subsequently, using a transgenic mouse expressing GFP under the EKLF promoter (pEKLF/ GFP; (Lohmann and Bieker, 2008)) it was shown that almost $30 \%$ of all $\mathrm{F} 4 / 80+$ macrophages from E13.5 FL are GFP+ (and thus likely EKLF+) (Xue et al., 2014). Indirect evidence (Li et al., 2019) demonstrated EKLF mRNA enrichment in Epor+F4/80+ macrophages that are more likely to form EBIs, indicating that EKLF expression is associated with EBI-forming macrophages. A direct analysis of RNA shows transient expression of EKLF in staged, sorted macrophage cells from the developing mouse FL (Mass et al., 2016). Finally, single cell RNA-Seq as well as flow cytometry directly show that a subpopulation of about $25 \%$ of total F4/80+ cells in E13.5 mouse FL express EKLF mRNA and protein (Mukherjee et al., 2021).

Earlier studies suggested that EKLF is important for the expression of DNase2a and Vcam1 in EBI macrophages (Porcu et al., 2011; Xue et al., 2014). However, given the severe alterations in EBIs from $\mathrm{EKLF}^{-1-}$ mice, and the known global role of EKLF in erythroid cells, it was unlikely that EKLF function in EBI macrophages would be restricted to just these two genes. To address whether EKLF also plays a global role in specifying gene expression in EBI macrophages one has to consider that since not all EBI macrophages express EKLF, one cannot simply study FL F4/80+ macrophage gene expression in $\mathrm{EKLF}^{-/-}$mice and derive definitive conclusions. An ideal system would be a conditional $\mathrm{EKLF}^{-/-}$in F4/80+ macrophages, but such a genetic system is not available as yet. Thus, in our study (Mukherjee et al., 2021) we combined gene expression data from two separate mouse genetic models to address this question.

First, we used the pEKLF/GFP mouse (Lohmann and Bieker, 2008) to separate EKLF/GFP+ and EKLF/GFP- F4/80+ macrophages from E13.5 mouse fetal liver and showed that, similar to Epor/eGFP+, only the EKLF/GFP+ macrophages are enriched for genes whose functions are relevant to the EBI niche macrophages. This includes iron homeostasis and transport, heme metabolism and erythroid differentiation (Mukherjee et al., 2021). Among the genes enriched in EKLF/GFP+ macrophages, 99 genes are also enriched in Epor/eGFP+ BM macrophages (Supplementary Table S2) and GO analysis of this gene set shows that they only contain genes involved in heme metabolism and iron homeostasis (Supplementary Table S1). The two RNA-Seq datasets were generated from very different erythropoiesis environments (adult BM vs embryonic FL), and thus these analyses show that EKLF+/Epor+ macrophages play a crucial role in providing iron and heme to developing erythroblasts at early stages of development and adult steady state erythropoiesis (Korolnek and Hamza, 2015; Ganz, 2016; Alam et al., 2017; Yeo et al., 2019a).

Second, RNA-Seq on F4/80+ macrophages isolated from $\mathrm{EKLF}^{+/+}$and $\mathrm{EKLF}^{-/-}$E13.5 FL shows that the predominant effect on gene expression in the absence of EKLF is gene downregulation (Mukherjee et al., 2021). This is consistent with the major role of EKLF as a transcription activator via its interaction with histone acetylases and other chromatin modifiers (Yien and Bieker, 2013), and seemingly EKLF activates the transcription of specific targets in F4/80+ macrophages. Further, about 500 genes that are significantly downregulated in $\mathrm{EKLF}^{-/-}$and enriched in EKLF/GFP+ macrophages are likely to be directly activated by EKLF binding to their promoters. This is supported by bioinformatic analysis showing that their promoters contain EKLF binding motifs (Mukherjee et al., 2021), but this in silico observation requires experimental validation. Binding motifs of other Klf family and E2f family TFs are also enriched in these promoters, and identification of these TFs here are reminiscent of a part of the EKLF-dependent transcriptional program in erythroid cells (Funnell et al., 2007; Eaton et al., 2008; Pilon et al., 2008; Tallack et al., 2009). This suggests the presence of either a transcription circuit or coregulators of transcription along with EKLF that may specify gene expression in EBI macrophages, but this again needs to be further validated by targeted genetic and molecular experiments to determine the specific roles of these TFs in EBI macrophages. In addition, other transcription factors in the EKLF-dependent gene expression program include MafK (NF-E2), Foxo3, Ikzf1, and Nr3c1(glucocorticoid receptor (Falchi et al., 2015)). It has been noted that KLF recognition motifs are enriched in active genes and are uniquely associated with CJUN 


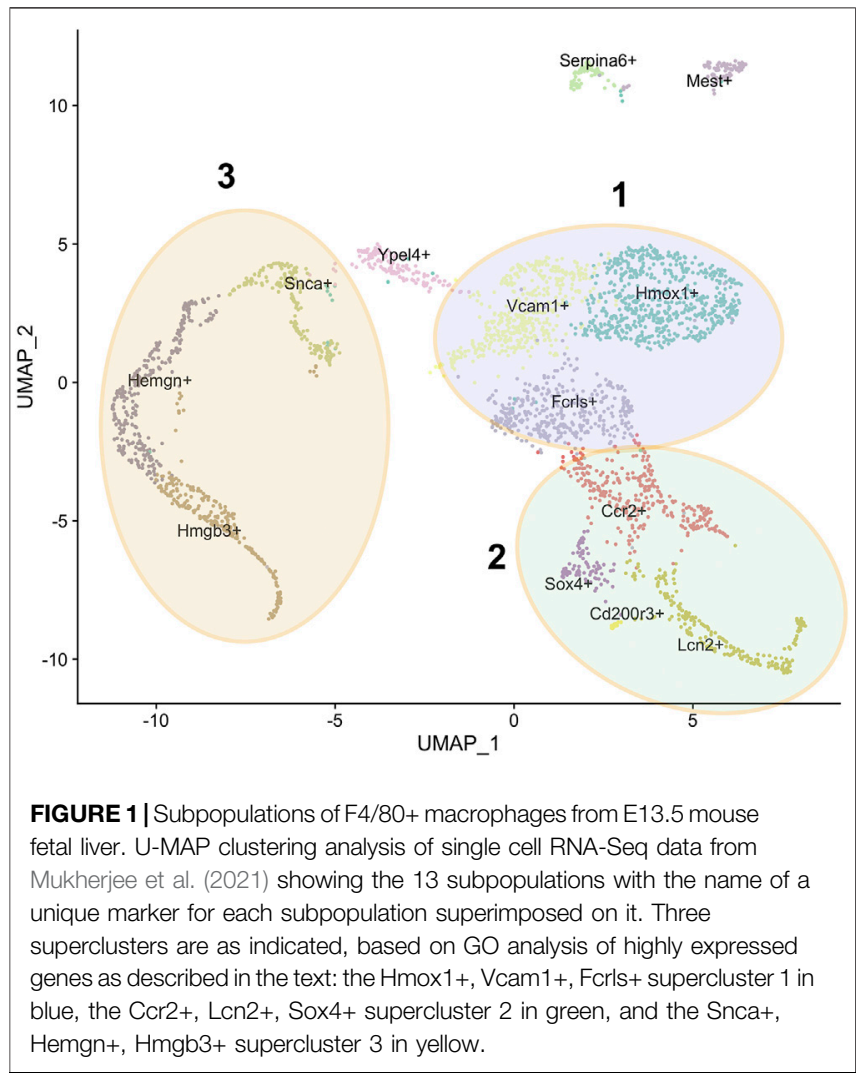

binding in genetically diverse mouse macrophage (Gosselin et al., 2014; Lavin et al., 2014; Link et al., 2018). These findings point to a definitive global role for EKLF in EBI macrophages, distinct from its erythroid role, but towards the same goal of ensuring efficient in vivo erythropoiesis.

\section{CELLULAR HETEROGENEITY IN E13.5 FL MACROPHAGES REVEALED BY SINGLE CELL RNA-SEQ}

The heterogeneity of macrophages in erythropoietic tissues has been apparent and efforts have attempted to resolve heterogeneity by assaying different cell surface markers associated with various subpopulations (Seu et al., 2017; Li et al., 2019; Tay et al., 2020). Even within the fraction of FL macrophages that form EBIs, there was evidence of heterogeneity due to distinct cell surface marker expression as well as varying morphology (Chasis and Mohandas, 2008; Manwani and Bieker, 2008; Yeo et al., 2016). Consistent with these studies, the Epor/eGFP and EKLF/GFP RNA-Seq datasets show at least two distinct populations in each case ( $\mathrm{Li}$ et al., 2019; Mukherjee et al., 2021) but firm conclusions about the extent of heterogeneity are difficult to draw from bulk RNA-Seq data. Single cell RNA-Seq (sc-seq) has been developed precisely for this purpose, and most researchers in the field were eager to apply sc-seq on EBI macrophages to resolve heterogeneity.

Our group published sc-seq data on $\mathrm{F} 4 / 80+$ macrophages isolated from E13.5 mouse FL (Mukherjee et al., 2021). A challenge that still persists is to decide upon a reliable marker for isolating EBI macrophages among the fraction of total macrophages and study only that fraction. We opted to use F4/80, which is expressed on the surface of all macrophages, and performed an unbiased survey of the extent of their heterogeneity based on single cell gene expression profiles. We applied unsupervised clustering and allowed for the maximum resolution of clusters based on principal component analysis. Our FL sc-seq shows more than 10 subpopulations with distinct gene expression profiles in each subpopulation corresponding to distinct functions and cell types. Further each cluster is associated with unique expression of at least one marker (Figure 1). We will elaborate further on the nature of the major subpopulations in greater detail in the following sections based on this sc-seq data (Mukherjee et al., 2021).

\section{CELLULAR PROPERTIES, MARKER EXPRESSION, AND FUNCTIONS OF VARIOUS FL MACROPHAGE SUBPOPULATIONS}

Most of the F4/80+ FL macrophages cluster near each other in the U-MAP of sc-seq data, apart from two small subpopulations marked by Serpina6 and Mest transcripts (Figure 1). All clusters express the Adgre 1 transcript that encodes the F4/80 protein, but each cluster expresses a variable amount (Mukherjee et al., 2021). This is consistent with the amount of F4/80 protein expression determined by Flow Cytometry that also shows variability in various cells. Based on GO analysis of the top significantly enriched genes in the minor Serpina6+ and Mest+ populations, it appears that the Serpina6+ population has enriched expression of genes involved in blood coagulation. Thus, these may be a small population of megakaryocyte lineage cells. Further, the Mest+ cluster likely comprises cells of mesodermal origin that are destined to form vasculature. Finally, the last subpopulation containing few cells is the Cd200r3+ population which comprise likely antigen-presenting macrophages that are enriched for genes involved in T-cell activation via antigen presentation.

Of the major populations, it is evident that there are three broad categories based on GO analysis of highly expressed genes. One comprises the Hmox1+, Vcam1+, Fcrls+ supercluster (Figure 1 - blue), second the Ccr2+, Lcn2+, Sox4+ supercluster (Figure 1 - green), and third the Snca+, Hemgn+, Hmgb3+ supercluster (Figure 1 - yellow). The Ypel4+ cluster has intermediate properties of superclusters 1 (Hmox1/Vcam1/Fcrls) and 3 (Snca/Hemgn/Hmgb3), as is also evidenced by its position in the U-MAP (Figure 1). Based on differentially expressed genes, the Hmox1+ and Vcam1+ subpopulations have a significant overlap in gene expression. These include endocytosis/cell import, cell adhesion, cell motility, iron homeostasis, cytoskeleton remodeling, innate immune response, myeloid leukocyte activation, cytokine production, and some metabolic processes. The Fcrls+ subpopulation also has similar properties as 


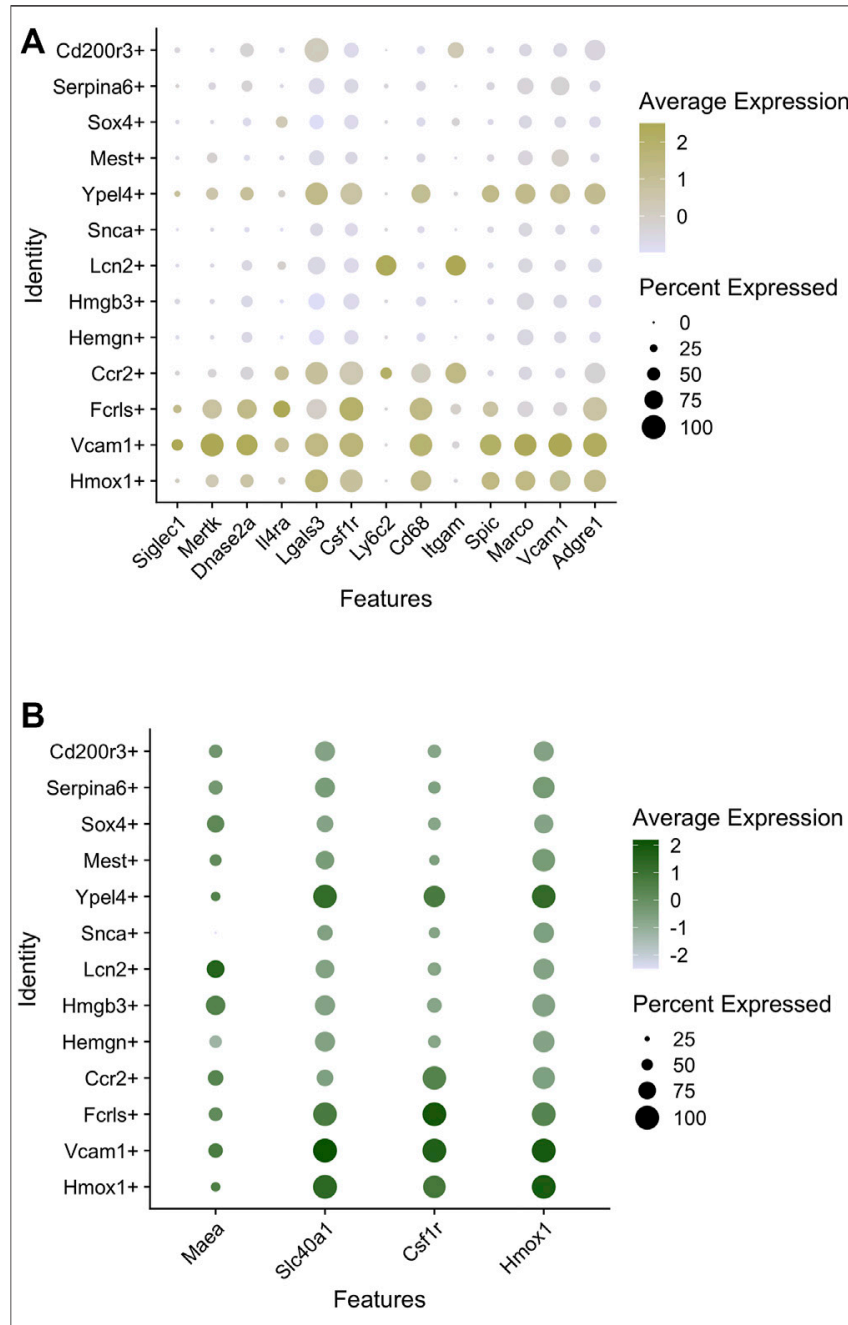

FIGURE 2| Macrophage marker expression in subpopulations of mouse E13.5 FL macrophages. (A) Bubble-plots showing the expression level of macrophage markers enriched in certain subpopulations. The intensity of the color indicates expression level of each gene whereas the size of the bubble depicts the relative fraction of cells in that subpopulation where the gene is expressed. (B) Bubble plots showing macrophage markers with similar levels of expression in most subpopulations.

the Vcam1+ subpopulation with notable common functions such as MAPK signaling, ERK1/2 signaling, IL-1 and IL-6 production, which are important for erythroblast maturation. Thus, it appears that the Hmox1+, Vcam1+, Fcrls+ clusters are at least partly comprised of macrophages that form the EBI niche, consistent with the fact that both Hmoxl and Vcam1 are known to be expressed in EBI macrophages (Sadahira et al., 1995; Fraser et al., 2015). In fact, on surveying known macrophage markers (that may or may not correlate with EBI niche formation), one finds that although many markers have similar expression in the Vcam1+ and Hmox1+ subpopulations (Figure 2A), the Vcam1+ subpopulation has significantly enriched expression of Dnase2a, Mertk, Siglec1 (Cd169) and Il4ra compared to the Hmox1+ subpopulation. Hmoxl expression itself is fairly ubiquitous in the isolated $\mathrm{F} 4 / 80+$ population, as is the expression of Slc40a1 (ferroportin) (Figure 2B), suggesting that expression of these genes may not be solely restricted to EBI macrophages.

In contrast, supercluster $2(\mathrm{Ccr} 2+/ \operatorname{Lcn} 2+/ \operatorname{Sox} 4+)$ is enriched for genes involved in innate immunity, $\mathrm{T}$-cell activation, cytotoxicity, leukocyte and lymphocyte differentiation, $\mathrm{T}$ and $\mathrm{B}$-cell development, suggesting that these subpopulations are similar to the Cd200r3 subpopulation also residing on the same arm of the U-MAP adjacent to these clusters. Therefore, these subpopulations of cells are likely comprised of activated macrophages that are geared towards provide innate immunity and assist in the development and differentiation of the adaptive immune response arm consisting of lymphocytes (Gautier et al., 2012; Lavin et al., 2014; Mass et al., 2016). One can rule out their involvement in forming the EBI niche.

The other arm of the U-MAP containing supercluster 3 (Snca+/Hemgn+/Hmgb3+) and the Ypel4+ subpopulation (Figure 1) is predominantly enriched for genes that are involved in erythroid and myeloid development, heme synthesis, and iron homeostasis, functions that are again attributable to the EBI niche (Korolnek and Hamza, 2015; Ganz, 2016; Alam et al., 2017; Yeo et al., 2019a). The Ypel4+ cluster is interesting since it not only expresses these genes, but also expresses genes involved in endocytosis, cell motility and cell adhesion indicating that this subpopulation of cells has some properties of the Hmox1+/Vcam1+ subpopulation as well. Indeed, this cluster is adjacent to the Vcam1+ cluster and expresses high levels of Vcam1 and Epor. The Hmgb3+ subpopulation seems to be composed of actively cycling cells with expression of genes involved in cell cycle, ribosome synthesis, protein synthesis, RNA processing and splicing. Supercluster 3 also express the EKLF/Klf1 transcription factor where Epor is partly enriched in the Snca+/Hemgn+ subpopulations. This is consistent with bulk RNA-seq data from our group as well as from Li et al. (2019) showing that EKLF+ macrophages are enriched for Epor expression and vice versa, but also demonstrating that there are unique subpopulations of EKLF and Epor expressing macrophages. It is tempting to speculate that the Hemgn+/Snca+ macrophage subpopulation expressing both EKLF and Epor form one subtype of EBI niche, whereas the Ypel4+/Vcam1+ subpopulations form another, but testing this hypothesis requires carefully crafted experiments.

On closer examination of the entire population for the expression of specific genes and cell surface markers of macrophages, we can detect 3 major patterns of marker expression (Figure 3A). We already found that Hmox1, Fpn (Slc40a1), Csf1r, and Maea ("pattern 1") are ubiquitously expressed in the whole population, and there is only a slight variation of expression in different subpopulations (Figure 3A). It would be interesting to determine the nature of this variability to determine cause-effect relationships between marker expression and heterogeneity. Additionally, we find that Vcam1 expression correlates highly with Axl, Timd4, Mertk, and Igf1 ("pattern 2"; Figure 3A), and all of these genes are known to be expressed in 


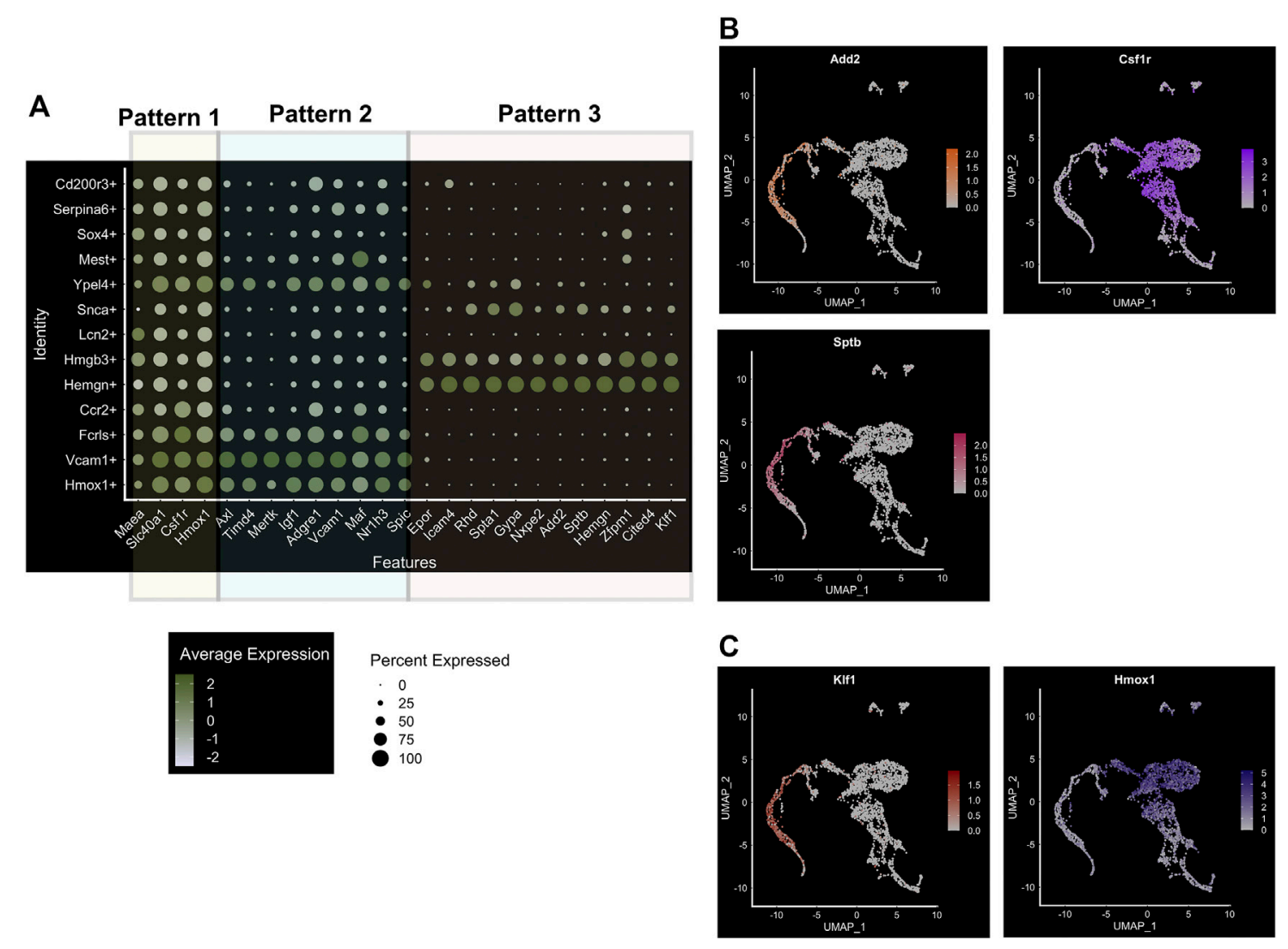

FIGURE 3 | Major subpopulations of mouse E13.5 FL macrophages. (A) Bubble-plot showing specific genes expressed in the three major superclusters of FL macrophages, including population-specific transcription factors. Three patterns are segregated as indicated, based on their common expression as expanded upon in the text. (B) Feature plot showing cell-surface marker expression associated with the superclusters 3 and 1, Sptb/Add2 and Csf1r, respectively. (C) Feature plot showing EKLF/KIf1 expression; compare to positive overlap with Sptb/Add2 in supercluster 3 (shown in (B)), but lack of overlap as compared to the cell-surface marker expression associated with supercluster 1 , Hmox 1.

subtypes of EBI niches (e.g., MerTK (Toda et al., 2014)). Finally, the third pattern of marker expression includes Epor, Gypa, Rhd, Icam4, Add2, and Sptb (Figure 3A). An interesting dichotomy is revealed from this analysis when we compare the expression of $A d d 2 / S p t b$ with Csf1r and find that they are expressed in mutually distinct superclusters (Figure 3B). Interestingly, these superclusters also correlate with EKLF/Klf1 and Hmox1 expression respectively (Figure 3C). Perhaps this is indicative of the heterogeneity seen within EBI macrophages with respect to gene expression and morphological features such as domed and flat (Yeo et al., 2016; Yeo et al., 2019b). Whether this may be due to different developmental origins or different stages of erythroid maturation of attached erythroblasts remains to be explored.

\section{SPECIFICATION OF HETEROGENEOUS CELL IDENTITY OF FL F4/80+ MACROPHAGES BY KEY TRANSCRIPTION FACTORS}

Our study focused primarily on the role of EKLF/Klf1 in FL macrophages, and hence we looked for cell surface markers of the
EKLF+ population, finding that Add2 (Adducin- $\beta$ ), Sptb ( $\beta$-spectrin), and Nxpe2 mRNA expression correlates very highly with EKLF and shows the same transient expression as EKLF (Mukherjee et al., 2021). We also demonstrated that Adducin- $\beta$ and $\beta$-spectrin proteins are expressed in a subpopulation of EBI macrophages. This confirms that the Hmgb3+/Hemgn+ subpopulations that are EKLF+ and Epor+ form at least one subtype of EBI macrophages. In addition, we find other cell surface markers enriched in the EKLF+ subpopulations but not correlating exclusively due to expression in the Ypel4 cluster, e.g., Gypa, Epor, Spta1, and Rhd (Figure 3A). The EKLF- subpopulations have enriched expression of the transcription factors Spi-C (Spic), Maf, and Nr1h3 (Figures 3C and 4A) each of which are implicated in EBI macrophage function (Kohyama et al., 2009; Kusakabe et al., 2011; Haldar et al., 2014; Zhao et al., 2021). We find that Klf1 and Spic/Maf/Nr1h3 expression are mutually exclusive, fitting into patterns 3 and 2 respectively (Figure $\mathbf{3 A}$ ). This suggests that these transcription factors drive transcription in distinct subpopulations of FL macrophages, and by extension, likely different categories of EBI macrophages. Exploring the direct transcriptional roles of EKLF, Spi-C, Maf and Nr1h3 could lead to 

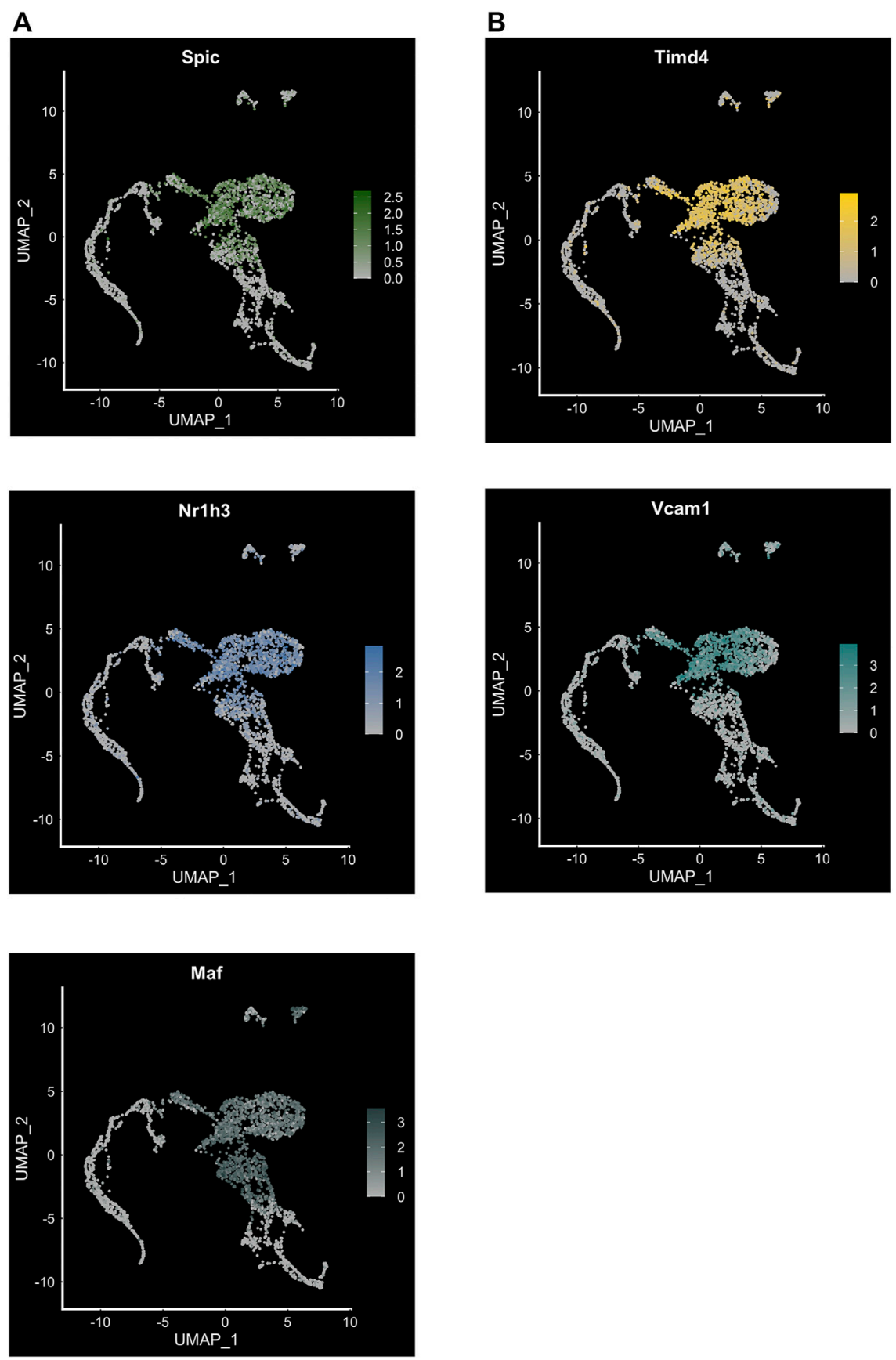

FIGURE 4 | Transcription factor expression and cell surface markers in FL macrophage subpopulations. (A) Feature plot showing expression of transcription factors Spic, Maf and Nr1h3 in the EKLF ${ }^{-/}$subpopulation of FL macrophages (compare to Figure $\mathbf{3 C}$ ). (B) Feature plot showing expression of cell surface markers Timd4 associated with Spi-C+, and Vcam1 associated with Maf+/Nr1h3+ subpopulations.

interesting insights into whether these subpopulations are also distinct in terms of morphology, developmental origin, and function in the EBI niche. In the context of bulk RNA-Seq studies (Li et al., 2019), it may appear surprising that Epor+ macrophages are enriched for EKLF/Klf1 as well as Spic, Maf, and $N r 1 h 3$, but this can be explained by the overlap in Epor and Spic/Maf/Nr1h3 expression in the Ypel4+ cluster as well as the Epor+ EKLF+ supercluster $($ Hmgb3+/Hemgn +$)$ (Figure 3A).

Similar to our approach for EKLF, we searched for cell surface markers that correlate with transcription factor expression in FL macrophages and find that Spic expression correlates highly with Timd 4 and that Maf and NrIh3 are most closely coexpressed with Vcam1 (Figures 4A and B). Consequently, to develop a viable 


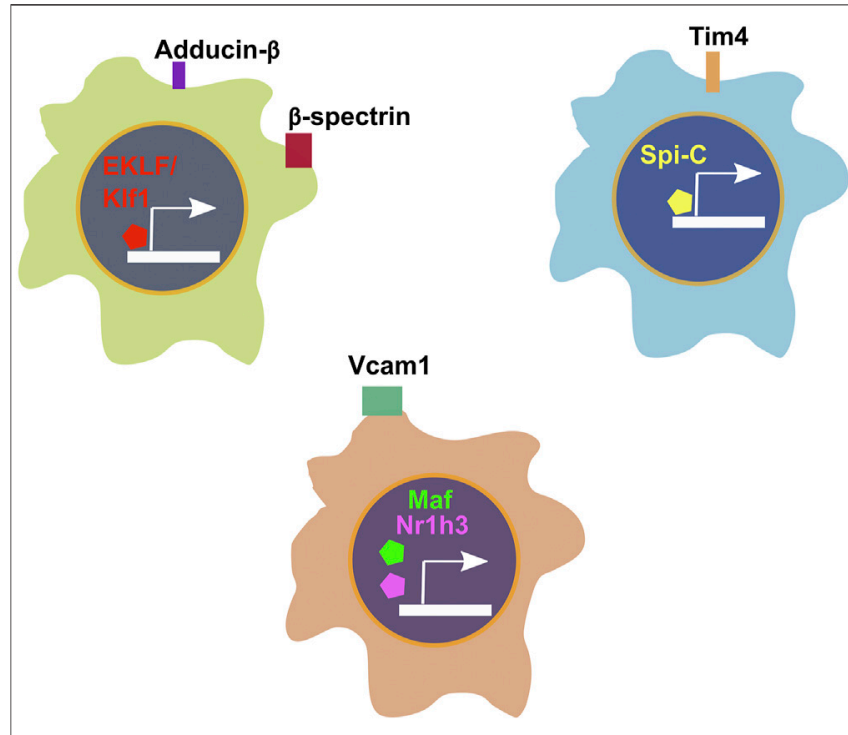

FIGURE 5 | Summary of transcription factors and corresponding cell surface markers associated with cellular heterogeneity in E13.5 FL macrophages.

strategy for separating the EKLF and Spi-C subpopulations, one approach would be to use the cell surface markers $S p t b / A d d 2$ and Timd4 or Vcam1 to separately isolate EKLF+ and Spi-C+/Maf+/ Nr1h3+ subpopulations for detailed characterization (Figure 5). As alluded to earlier, it would be incredibly interesting to determine whether these starkly distinct populations have separate developmental origins but convergent functions in erythroid development. It is also likely that both subpopulations form EBI niches, but in different contexts depending on the stage of maturation of the attached erythroid progenitors.

\section{SUMMARY AND OUTSTANDING QUESTIONS}

From earlier studies focusing on morphology and cell surface marker expression in EBI macrophages in various hematopoietic tissues, to the current attempts in determining gene expression at bulk and single cell resolution in various tissues, the characterization of EBI macrophages have come a long way. However, there is much to learn about this intriguing cell-type in all the above areas. Despite earlier efforts, we still lack clarity on the exact nature and types of EBI macrophages based on cell surface marker expression, and as a result, we still lack a coherent strategy for isolation of various EBI macrophage subpopulations to study their form and function. Population heterogeneity continues to complicate interpretations of data generated using F4/80+ macrophages. In addition and as previously discussed (Mukherjee et al., 2021), different subtypes may dynamically appear under stress or pathological conditions, and specific expression patterns may also depend on the niche environment (fetal liver vs bone marrow vs spleen (Gosselin et al., 2014; Lavin et al., 2014)) and modified by neural signaling in the bone marrow (Mendez-Ferrer et al., 2020). These may give rise to morphologically distinct classes of islands as observed by electron microscopy (Yeo et al., 2016).

In this context, one should avoid confusion between use of a protein as a marker versus its functional requirement for the cell. The suggestion that Vcam1 expression in macrophage is not required for erythroblastic island formation (Wei et al., 2019) does not negate its usefulness as a selection marker of a subset of islands. As another example, deletion of the widely-used F4/80 enrichment marker has no effect on macrophage viability/ function (Schaller et al., 2002).

An additional challenge in the field has been the concern that pieces of macrophage can contaminate other cell types and have a confounding effect on conclusions from mRNA expression analysis. Contamination is apparent when analyzing flowsorted single cells by image stream, where punctate expression from macrophage remnants attached at the cell surface are visible (Levesque et al., 2021). However, the extensive efforts to morphologically verify sorted single cell isolates, the inclusion of small peptides during isolation that disrupt erythroid/ macrophage interactions, the verification of homogeneous distribution of marker expression in single cell images, and the clear segregation of cell surface expression used in the recent studies (Li et al., 2019; Mukherjee et al., 2021; Zhang et al., 2021) alleviate contamination concerns.

It is hoped the single cell studies provide foundation for breakthroughs in understanding heterogeneity and function by validation of FACS-based isolation methods for various subpopulations of $\mathrm{F} 4 / 80+$ macrophages. However, some outstanding questions remain:

\section{Developmental Onset of Critical Cell Types}

There remains considerable ambiguity regarding what inherent cellular properties or external factors drive EBI niche formation by macrophages. In this context, recent studies implicating PPAR $\gamma$ in EBI development have opened up a window into the potential non-lipid-dependent role of this transcription factor in the adult bone marrow and spleen, perhaps by its modulation of macrophage subset lineage decisions and establishing identity (Okreglicka et al., 2021). Additionally, structural proteins such as tropomodulin3 are implicated in both erythroid and macrophage coordinate function in the EBI (Sui et al., 2014). However, in essence we know little about developmental origins of macrophages destined to form the EBI niche, and it appears from our single cell studies that there might be distinct populations of such macrophages likely originating from alternative sources. It is exciting to note that these subpopulations also express unique transcription factors, and this opens up multiple avenues of exploration (Figure 5). One interesting approach would be to examine how EKLF expression in these subpopulations is initiated and regulated. Further, one must determine how EKLF function in macrophages differs from its erythroid counterpart; since EKLF has a conserved DNA binding sequence (5'CCMCRCCCN; (Tallack et al., 2010; Gillinder et al., 2017; Kulczynska et al., 2020)), what prevents EKLF binding to erythroid targets and driving the erythroid gene 
program in macrophages? One likelihood is that EKLF has separate molecular interactions with transcription cofactors leading to distinct chromatin dynamics at EKLF targets in erythroid cells and macrophages, perhaps determined by its cell- or stage-specific post-translational modification status (discussed in Yien and Bieker (2013)).

\section{Alternate Island Populations}

We have not addressed other cell types that may also be playing a role in the island niche. For example, the Kalfa lab analyzed single cells derived from isolated whole erythroblastic islands (Seu et al., 2018). Their data support the idea that non-F4/80 cells (ie, F4/80Ly6G+ granulocytes) may play a role in a separate subset of island function, particularly as related to myelopoiesis following anemia of inflammation, leading to a dynamic antagonistic balance between granulopoiesis and erythropoiesis, a notion supported by the dramatic effects of G-CSF treatment on suppression of erythropoiesis (Jacobsen et al., 2014; Jacobsen et al., 2015). These data are consistent with imaging multi-color fluorescence analyses showing that F4/80, VCAM1, and CD169 (Siglec1), but not CD11b (Itgam) or Ly6G, are expressed in erythroblastic islands, while at the same time, the CD11b+Ly6G+F4/80 - cells can be found "at the periphery" of a subset of these aggregates (Tay et al., 2020). The dynamic balance between these populations in the bone marrow versus the spleen, and whether they reside in the same 'niche' or are separate entities, will be quite interesting to quantify and delineate.

\section{Role of EKLF Mutations in Erythroblastic Island Macrophage Alterations}

Two dominant monoallelic mutations in mammalian EKLF are causative for hematologic deficiencies: the mouse Nan mutation (E339D) leads to hemolytic neonatal anemia, and the human CDA mutation (E325K) causes congenital dyserythropoietic anemia (CDA) type IV (reviewed in Kulczynska-Figurny et al. (2020)). Although numerous other monoallelic mutations have been identified that lead to haploinsufficiency (Waye and Eng, 2015; Perkins et al., 2016), these have mild phenotypic effects. However, inheritance of two altered alleles can lead to compound heterozygosity and effects on blood cell production and properties, leading to anemia (Perkins et al., 2016; Tangsricharoen et al., 2021; Xu et al., 2021). These analyses have completely focused on effect on the red cell; however, it is easily conceivable that the island macrophage is also affected, particularly as the Nan and CDA mutations alter EKLF DNA recognition properties that lead to neomorphic expression changes and systemic effects (Kulczynska-Figurny et al., 2020).

\section{Relevance to Human Biology}

Human macrophage also exhibit an extensive level of heterogeneity during development (Bian et al., 2020). However, many of the same subtype expression patterns described here for the mouse are also apparent from single cell analysis of human fetal liver cells, particularly within the "erythroblastic island macrophage" cluster (Popescu et al., 2019). In addition, a role for EKLF in proper programming of macrophage for island function is suggested by gain-of-function studies in macrophage derived from human iPS cells (Lopez-Yrigoyen et al., 2019). There remains a need to increase the expansion capability of erythroid cells in culture for their clinical use (Migliaccio et al., 2012; Heshusius et al., 2019; Olivier et al., 2019; Deleschaux et al., 2020; Pellegrin et al., 2021), and identification of the relevant macrophage subtype can inform approaches to overcome heterogeneity and utilize the optimal cells to aid efficient in vitro erythropoiesis (Rhodes et al., 2008; Hom et al., 2015; May and Forrester, 2020). Indeed, human island macrophage function can be recapitulated in culture by inclusion of lipids and by glucocorticoid receptor activation (Falchi et al., 2015; Heideveld et al., 2018).

\section{FINAL COMMENTS}

Establishing the necessity of EBI macrophage function for in vivo erythropoiesis remains challenging since contingency mechanisms have evolved to compensate for their loss. Future work in this area must aim to deplete not only EBI macrophages but also monocytes, but that can lead to other complications with immune function, unrelated to erythropoiesis. Still, the exact physiological role of EBIs in erythropoiesis at different stages of development, in different hematopoietic tissues, and in the context of diseases such as anemia and dysplasia, remains to be extensively characterized. Overall, the study of EBI macrophages is at an exciting juncture where the progress that has been made in gene expression studies can be extended into various erythropoietic tissues and conditions, and used as a guide for developing hypotheses to characterize their molecular nature and developmental origins.

\section{AUTHOR CONTRIBUTIONS}

KM performed analyses; KM and JJB wrote the manuscript.

\section{FUNDING}

This work was supported by a Black Family Stem Cell Institute postdoctoral award to KM and by NIH grant R01 DK121671 to JJB.

\section{SUPPLEMENTARY MATERIAL}

The Supplementary Material for this article can be found online at: https://www.frontiersin.org/articles/10.3389/fgene.2021.756028/ full\#supplementary-material 


\section{REFERENCES}

Alam, M. Z., Devalaraja, S., and Haldar, M. (2017). The Heme Connection: Linking Erythrocytes and Macrophage Biology. Front. Immunol. 8, 33. doi:10.3389/ fimmu.2017.00033

Bernard, J. (1991). The Erythroblastic Island: Past and Future. Blood Cells 17 (1), 5-4.

Bessis, M. (1958). Erythroblastic Island, Functional unity of Bone Marrow. Rev. Hematol. 13 (1), 8-11. doi:10.1182/blood.v13.4.410.410

Bian, Z., Gong, Y., Huang, T., Lee, C. Z. W., Bian, L., Bai, Z., et al. (2020). Deciphering Human Macrophage Development at Single-Cell Resolution. Nature 582 (7813), 571-576. doi:10.1038/s41586-020-2316-7

Bieker, J. J. (2010). Putting a finger on the Switch. Nat. Genet. 42 (9), 733-734. doi:10.1038/ng0910-733

Buesche, G., Teoman, H., Giagounidis, A., Gohring, G., Schlegelberger, B., Ganser, A., et al. (2016). Impaired Formation of Erythroblastic Islands Is Associated with Erythroid Failure and Poor Prognosis in a Significant Proportion of Patients with Myelodysplastic Syndromes. Haematologica 101 (5), e177-e181. doi:10.3324/haematol.2015.129015

Cao, S., Liu, J., Song, L., and Ma, X. (2005). The Protooncogene C-Maf Is an Essential Transcription Factor for IL-10 Gene Expression in Macrophages. J. Immunol. 174 (6), 3484-3492. doi:10.4049/jimmunol.174.6.3484

Chasis, J. A., and Mohandas, N. (2008). Erythroblastic Islands: Niches for Erythropoiesis. Blood 112 (3), 470-478. doi:10.1182/blood-2008-03-077883

Chen, Y., Xiang, J., Qian, F., Diwakar, B. T., Ruan, B., Hao, S., et al. (2020). Epo Receptor Signaling in Macrophages Alters the Splenic Niche to Promote Erythroid Differentiation. Blood 136 (2), 235-246. doi:10.1182/ blood.2019003480

Chow, A., Huggins, M., Ahmed, J., Hashimoto, D., Lucas, D., Kunisaki, Y., et al. (2013). CD169+ Macrophages Provide a Niche Promoting Erythropoiesis under Homeostasis and Stress. Nat. Med. 19 (4), 4293057-4293436. doi:10.1038/nm.3057

de Back, D. Z., Kostova, E. B., van Kraaij, M., van den Berg, T. K., and van Bruggen, R. (2014). Of Macrophages and Red Blood Cells; A Complex Love story. Front. Physiol. 5, 9. doi:10.3389/fphys.2014.00009

Deleschaux, C., Moras, M., Lefevre, S. D., and Ostuni, M. A. (2020). An Overview of Different Strategies to Recreate the Physiological Environment in Experimental Erythropoiesis. Int. J. Mol. Sci. 21 (15), 5263. doi:10.3390/ijms21155263

Eaton, S. A., Funnell, A. P. W., Sue, N., Nicholas, H., Pearson, R. C. M., and Crossley, M. (2008). A Network of Krüppel-like Factors (Klfs). J. Biol. Chem. 283 (40), 26937-26947. doi:10.1074/jbc.m804831200

Falchi, M., Varricchio, L., Martelli, F., Masiello, F., Federici, G., Zingariello, M., et al. (2015). Dexamethasone Targeted Directly to Macrophages Induces Macrophage Niches that Promote Erythroid Expansion. Haematologica 100 (2), 178-187. doi:10.3324/haematol.2014.114405

Fraser, S. T., Midwinter, R. G., Coupland, L. A., Kong, S., Berger, B. S., Yeo, J. H., et al. (2015). Heme Oxygenase-1 Deficiency Alters Erythroblastic Island Formation, Steady-State Erythropoiesis and Red Blood Cell Lifespan in Mice. Haematologica 100 (5), 601-610. doi:10.3324/haematol.2014.116368

Funnell, A. P. W., Maloney, C. A., Thompson, L. J., Keys, J., Tallack, M., Perkins, A. C., et al. (2007). Erythroid Krüppel-like Factor Directly Activates the Basic Krüppel-like Factor Gene in Erythroid Cells. Mol. Cell Biol 27 (7), 2777-2790. doi:10.1128/mcb.01658-06

Ganz, T. (2016). Macrophages and Iron Metabolism. Microbiol. Spectr. 4 (5), MCHD-0037-2016. doi:10.1128/microbiolspec.MCHD-0037-2016

Gautier, E. L., Shay, T., Shay, T., Miller, J., Greter, M., Jakubzick, C., et al. (2012). Gene-expression Profiles and Transcriptional Regulatory Pathways that Underlie the Identity and Diversity of Mouse Tissue Macrophages. Nat. Immunol. 13 (11), 1118-1128. doi:10.1038/ni.2419

Gillinder, K. R., Ilsley, M. D., Nébor, D., Sachidanandam, R., Lajoie, M., Magor, G. W., et al. (2017). Promiscuous DNA-Binding of a Mutant Zinc finger Protein Corrupts the Transcriptome and Diminishes Cell Viability. Nucleic Acids Res. 45 (3), 1130-1143. doi:10.1093/nar/gkw1014

Gnanapragasam, M. N., and Bieker, J. J. (2017). Orchestration of Late Events in Erythropoiesis by KLF1/EKLF. Curr. Opin. Hematol. 24 (3), 183-190. doi:10.1097/MOH.0000000000000327
Gosselin, D., Link, V. M., Romanoski, C. E., Fonseca, G. J., Eichenfield, D. Z., Spann, N. J., et al. (2014). Environment Drives Selection and Function of Enhancers Controlling Tissue-specific Macrophage Identities. Cell 159 (6), 1327-1340. doi:10.1016/j.cell.2014.11.023

Haldar, M., Kohyama, M., So, A. Y.-L., Kc, W., Wu, X., Briseño, C. G., et al. (2014). Heme-mediated SPI-C Induction Promotes Monocyte Differentiation Into Iron-Recycling Macrophages. Cell 156 (6), 1223-1234. doi:10.1016/ j.cell.2014.01.069

Heideveld, E., Hampton-O’Neil, L. A., Cross, S. J., van Alphen, F. P. J., van den Biggelaar, M., Toye, A. M., et al. (2018). Glucocorticoids Induce Differentiation of Monocytes towards Macrophages that Share Functional and Phenotypical Aspects with Erythroblastic Island Macrophages. Haematologica 103 (3), 395-405. doi:10.3324/haematol.2017.179341

Heshusius, S., Heideveld, E., Burger, P., Thiel-Valkhof, M., Sellink, E., Varga, E., et al. (2019). Large-scale In Vitro Production of Red Blood Cells from Human Peripheral Blood Mononuclear Cells. Blood Adv. 3 (21), 3337-3350. doi:10.1182/bloodadvances.2019000689

Hom, J., Dulmovits, B. M., Mohandas, N., and Blanc, L. (2015). The Erythroblastic Island as an Emerging Paradigm in the Anemia of Inflammation. Immunol. Res. 63 (1-3), 75-89. doi:10.1007/s12026-015-8697-2

Jacobsen, R. N., Forristal, C. E., Raggatt, L. J., Nowlan, B., Barbier, V., Kaur, S., et al. (2014). Mobilization with Granulocyte Colony-Stimulating Factor Blocks Medullar Erythropoiesis by Depleting F4/80+VCAM1+CD169+ERHR3+Ly6G+ Erythroid Island Macrophages in the Mouse. Exp. Hematol. 42 (7), 547-5618. doi:10.1016/j.exphem.2014.03.009

Jacobsen, R. N., Perkins, A. C., and Levesque, J.-P. (2015). Macrophages and Regulation of Erythropoiesis. Curr. Opin. Hematol. 22 (3), 212-219. doi:10.1097/MOH.0000000000000131

Klei, T. R. L., Meinderts, S. M., van den Berg, T. K., and van Bruggen, R. (2017). From the Cradle to the Grave: The Role of Macrophages in Erythropoiesis and Erythrophagocytosis. Front. Immunol. 8, 73. doi:10.3389/fimmu.2017.00073

Kohyama, M., Ise, W., Edelson, B. T., Wilker, P. R., Hildner, K., Mejia, C., et al. (2009). Role for Spi-C in the Development of Red Pulp Macrophages and Splenic Iron Homeostasis. Nature 457 (7227), 318-321. doi:10.1038/ nature 07472

Korolnek, T., and Hamza, I. (2015). Macrophages and Iron Trafficking at the Birth and Death of Red Cells. Blood 125 (19), 2893-2897. doi:10.1182/blood-2014$12-567776$

Koury, M. J. (2014). Abnormal Erythropoiesis and the Pathophysiology of Chronic Anemia. Blood Rev. 28 (2), 49-66. doi:10.1016/j.blre.2014.01.002

Kulczynska, K., Bieker, J. J., and Siatecka, M. (2020). A Krüppel-like Factor 1 (KLF1) Mutation Associated with Severe Congenital Dyserythropoietic Anemia Alters its DNA-Binding Specificity. Mol. Cell Biol. 40 (5), e00444-19. doi:10.1128/MCB.00444-19

Kulczynska-Figurny, K., Bieker, J. J., and Siatecka, M. (2020). Severe Anemia Caused by Dominant Mutations in Krüppel-like Factor 1 (KLF1). Mutat. Res. Rev. Mutat. Res. 786, 108336. doi:10.1016/j.mrrev.2020.108336

Kusakabe, M., Hasegawa, K., Hamada, M., Nakamura, M., Ohsumi, T., Suzuki, H., et al. (2011). c-Maf Plays a Crucial Role for the Definitive Erythropoiesis that Accompanies Erythroblastic Island Formation in the Fetal Liver. Blood 118 (5), 1374-1385. doi:10.1182/blood-2010-08-300400

Lavin, Y., Winter, D., Blecher-Gonen, R., David, E., Keren-Shaul, H., Merad, M., et al. (201401449). Tissue-resident Macrophage Enhancer Landscapes are Shaped by the Local Microenvironment. Cell 159 (614), 1312-13264. doi:10.1016/j.cell.2014.11.018

Levesque, J.-P., Summers, K. M., Bisht, K., Millard, S. M., Winkler, I. G., and Pettit, A. R. (2021). Macrophages Form Erythropoietic Niches and Regulate Iron Homeostasis to Adapt Erythropoiesis in Response to Infections and Inflammation. Exp. Hematol., S0301-472x(21)00291-5, 2021 [Epub ahead of print]. doi:10.1016/j.exphem.2021.08.011

Li, W., Guo, R., Song, Y., and Jiang, Z. (2020). Erythroblastic Island Macrophages Shape Normal Erythropoiesis and Drive Associated Disorders in Erythroid Hematopoietic Diseases. Front. Cell Dev. Biol. 8, 613885. doi:10.3389/ fcell.2020.613885

Li, W., Wang, Y., Zhao, H., Zhang, H., Xu, Y., Wang, S., et al. (2019). Identification and Transcriptome Analysis of Erythroblastic Island Macrophages. Blood 134, 480-491. doi:10.1182/blood.2019000430 
Liao, C., Prabhu, K. S., and Paulson, R. F. (2018). Monocyte-derived Macrophages Expand the Murine Stress Erythropoietic Niche during the Recovery from Anemia. Blood 132 (24), 2580-2593. doi:10.1182/blood-2018-06-856831

Lifshitz, L., Tabak, G., Gassmann, M., Mittelman, M., and Neumann, D. (2010). Macrophages as Novel Target Cells for Erythropoietin. Haematologica 95 (11), 1823-1831. doi:10.3324/haematol.2010.025015

Link, V. M., Duttke, S. H., Chun, H. B., Holtman, I. R., Westin, E., Hoeksema, M. A., et al. (2018). Analysis of Genetically Diverse Macrophages Reveals Local and Domain-wide Mechanisms that Control Transcription Factor Binding and Function. Cell 173 (7), 1796-1809. doi:10.1016/j.cell.2018.04.018

Lohmann, F., and Bieker, J. J. (2008). Activation of Eklf Expression during Hematopoiesis by Gata2 and Smad5 Prior to Erythroid Commitment. Development 135 (12), 2071-2082. doi:10.1242/dev.018200

Lopez-Yrigoyen, M., Yang, C.-T., Fidanza, A., Cassetta, L., Taylor, A. H., McCahill, A., et al. (2019). Genetic Programming of Macrophages Generates an In Vitro Model for the Human Erythroid Island Niche. Nat. Commun. 10 (1), 881. doi:10.1038/s41467-019-08705-0

Luo, B., Gan, W., Liu, Z., Shen, Z., Wang, J., Shi, R., et al. (2016). Erythropoeitin Signaling in Macrophages Promotes Dying Cell Clearance and Immune Tolerance. Immunity 44 (2), 287-302. doi:10.1016/j.immuni.2016.01.002

Manwani, D., and Bieker, J. J. (2008). Chapter 2 the Erythroblastic Island. Curr. Top. Dev. Biol. 82, 23-53. doi:10.1016/s0070-2153(07)00002-6

Mass, E., Ballesteros, I., Farlik, M., Halbritter, F., Günther, P., Crozet, L., et al. (2016). Specification of Tissue-Resident Macrophages During Organogenesis. Science 353 (6304), aaf4238. doi:10.1126/science.aaf 4238

May, A., and Forrester, L. M. (2020). The Erythroblastic Island Niche: Modeling in Health, Stress, and Disease. Exp. Hematol. 91, 10-21. doi:10.1016/ j.exphem.2020.09.185

Méndez-Ferrer, S., Bonnet, D., Steensma, D. P., Hasserjian, R. P., Ghobrial, I. M., Gribben, J. G., et al. (2020). Bone Marrow Niches in Haematological Malignancies. Nat. Rev. Cancer 20 (5), 285-298. doi:10.1038/s41568-020$0245-2$

Migliaccio, A. R., Whitsett, C., Papayannopoulou, T., and Sadelain, M. (2012). The Potential of Stem Cells as an In Vitro Source of Red Blood Cells for Transfusion. Cell Stem Cell 10 (2), 115-119. doi:10.1016/j.stem.2012.01.001

Mohandas, N., and Prenant, M. (1978). Three-Dimensional Model of Bone Marrow. Blood 51 (4), 633-643. doi:10.1182/blood.v51.4.633.633

Mukherjee, K., Xue, L., Planutis, A., Gnanapragasam, M. N., Chess, A., and Bieker, J. J. (2021). EKLF/KLF1 Expression Defines a Unique Macrophage Subset during Mouse Erythropoiesis. Elife 10. doi:10.7554/eLife.61070

Nakamura, M., Hamada, M., Hasegawa, K., Kusakabe, M., Suzuki, H., Greaves, D. R., et al. (2009). c-Maf is Essential for the F4/80 Expression in Macrophages In Vivo. Gene 445 (1-2), 66-72. doi:10.1016/j.gene.2009.06.003

Okreglicka, K., Iten, I., Pohlmeier, L., Onder, L., Feng, Q., Kurrer, M., et al. (2021). $\operatorname{PPAR} \gamma$ Is Essential for the Development of Bone Marrow Erythroblastic Island Macrophages and Splenic Red Pulp Macrophages. J. Exp. Med. 218 (5). doi:10.1084/jem.20191314

Olivier, E. N., Zhang, S., Yan, Z., Suzuka, S., Roberts, K., Wang, K., et al. (2019). PSC-RED and MNC-RED: Albumin-free and Low-Transferrin Robust Erythroid Differentiation Protocols to Produce Human Enucleated Red Blood Cells. Exp. Hematol. 75, 31-52. doi:10.1016/j.exphem.2019.05.006

Paulson, R. F. (2019). Epo Receptor marks the Spot. Blood 134 (5), 413134-413414. doi:10.1182/blood.2019001581

Pellegrin, S., Severn, C. E., and Toye, A. M. (2021). Towards Manufactured Red Blood Cells for the Treatment of Inherited Anemia. Haematologica 106, 2304-2311. doi:10.3324/haematol.2020.268847

Perkins, A., Xu, X., Higgs, D. R., Patrinos, G. P., Arnaud, L., Bieker, J. J., et al. (2016). Krüppeling Erythropoiesis: an Unexpected Broad Spectrum of Human Red Blood Cell Disorders Due to KLF1 Variants. Blood 127 (15), 1856-1862. doi:10.1182/blood-2016-01-694331

Pilon, A. M., Arcasoy, M. O., Dressman, H. K., Vayda, S. E., Maksimova, Y. D., Sangerman, J. I., et al. (2008). Failure of Terminal Erythroid Differentiation in EKLF-Deficient Mice Is Associated with Cell Cycle Perturbation and Reduced Expression of E2F2. Mol. Cell Biol. 28 (24), 7394-7401. doi:10.1128/ mcb.01087-08

Popescu, D.-M., Botting, R. A., Stephenson, E., Green, K., Webb, S., Jardine, L., et al. (2019). Decoding Human Fetal Liver Haematopoiesis. Nature 574 (7778), 365-371. doi:10.1038/s41586-019-1652-y
Porcu, S., Manchinu, M. F., Marongiu, M. F., Sogos, V., Poddie, D., Asunis, I., et al. (2011). Klf1 Affects DNase II-Alpha Expression in the central Macrophage of a Fetal Liver Erythroblastic Island: A Non-Cell-Autonomous Role in Definitive Erythropoiesis. Mol. Cell Biol. 31 (19), 4144-4154. doi:10.1128/mcb.05532-11

Ramos, P., Casu, C., Gardenghi, S., Breda, L., Crielaard, B. J., Guy, E., et al. (2013). Macrophages Support Pathological Erythropoiesis in Polycythemia Vera and $\beta$-Thalassemia. Nat. Med. 19 (4), 437-445. doi:10.1038/nm.3126

Rhodes, M. M., Kopsombut, P., Bondurant, M. C., Price, J. O., and Koury, M. J. (2008). Adherence to Macrophages in Erythroblastic Islands Enhances Erythroblast Proliferation and Increases Erythrocyte Production by a Different Mechanism Than Erythropoietin. Blood 111 (3), 1700-1708. doi:10.1182/blood-2007-06-098178

Sadahira, Y., Yasuda, T., and Kimoto, T. (1991). Regulation of Forssman Antigen Expression during Maturation of Mouse Stromal Macrophages in Haematopoietic Foci. Immunology 73 (4), 498-504.

Sadahira, Y., Yasuda, T., Yoshino, T., Manabe, T., Takeishi, T., Kobayashi, Y., et al. (2000). Impaired Splenic Erythropoiesis in Phlebotomized Mice Injected with CL2MDP-Liposome: An Experimental Model for Studying the Role of Stromal Macrophages in Erythropoiesis. J. Leukoc. Biol. 68 (4), 464-470.

Sadahira, Y., Yoshino, T., and Monobe, Y. (1995). Very Late Activation Antigen 4vascular Cell Adhesion Molecule 1 Interaction is Involved in the Formation of Erythroblastic Islands. J. Exp. Med. 181 (1), 411-415. doi:10.1084/jem.181.1.411

Schaller, E., Macfarlane, A. J., Rupec, R. A., Gordon, S., McKnight, A. J., and Pfeffer, K. (2002). Inactivation of the F4/80 Glycoprotein in the Mouse Germ Line. Mol. Cell Biol 22 (22), 8035-8043. doi:10.1128/MCB.22.22.8035-8043.2002

Seu, K. G., Papoin, J., Fessler, R., Hom, J., Huang, G., Mohandas, N., et al. (2017). Unraveling Macrophage Heterogeneity in Erythroblastic Islands. Front. Immunol. 8, 1140. doi:10.3389/fimmu.2017.01140

Seu, K. G., Romano, L., Papoin, J., Muench, E. D., Konstantinidis, D., Olsson, A., et al. (2018). "The Erythro-Myeloblastic Island (EMBI): A Hematopoietic Niche Balancing Erythropoiesis and Myelopopoiesis," in American Society for Hematology Annual Meeting, San Diego, CA, December 3, 2018 (Washington, DC: American Society of Hematology).

Siatecka, M., and Bieker, J. J. (2011). The Multifunctional Role of EKLF/KLF1 during Erythropoiesis. Blood 118 (8), 2044-2054. doi:10.1182/blood-2011-03-331371

Soni, S., Bala, S., Gwynn, B., Sahr, K. E., Peters, L. L., and Hanspal, M. (2006). Absence of Erythroblast Macrophage Protein (Emp) Leads to Failure of Erythroblast Nuclear Extrusion. J. Biol. Chem. 281 (29), 20181-20189. doi:10.1074/jbc.m603226200

Sui, Z., Nowak, R. B., Bacconi, A., Kim, N. E., Liu, H., Li, J., et al. (2014). Tropomodulin3-null Mice Are Embryonic Lethal with Anemia Due to Impaired Erythroid Terminal Differentiation in the Fetal Liver. Blood 123 (5), 758-767. doi:10.1182/blood-2013-03-492710

Tallack, M. R., Keys, J. R., Humbert, P. O., and Perkins, A. C. (2009). EKLF/KLF1 Controls Cell Cycle Entry via Direct Regulation of E2f2. J. Biol. Chem. 284 (31), 20966-20974. doi:10.1074/jbc.m109.006346

Tallack, M. R., and Perkins, A. C. (2010). KLF1 Directly Coordinates Almost All Aspects of Terminal Erythroid Differentiation. IUBMB Life 62 (12), 886-890. doi:10.1002/iub.404

Tallack, M. R., Whitington, T., Shan Yuen, W., Wainwright, E. N., Keys, J. R., Gardiner, B. B., et al. (2010). A Global Role for KLF1 in Erythropoiesis Revealed by ChIP-Seq in Primary Erythroid Cells. Genome Res. 20 (8), 1052-1063. doi:10.1101/gr.106575.110

Tangsricharoen, T., Natesirinilkul, R., Phusua, A., Fanhchaksai, K., Ittiwut, C., Chetruengchai, W., et al. (2021). Severe Neonatal Haemolytic Anaemia Caused by Compound Heterozygous KLF1 Mutations: Report of Four Families and Literature Review. Br. J. Haematol. 194, 626-634. doi:10.1111/bjh.17616

Tay, J., Bisht, K., McGirr, C., Millard, S. M., Pettit, A. R., Winkler, I. G., et al. (2020). Imaging Flow Cytometry Reveals that Granulocyte colony-stimulating Factor Treatment Causes Loss of Erythroblastic Islands in the Mouse Bone Marrow. Exp. Hematol. 82, 33-42. doi:10.1016/j.exphem.2020.02.003

Toda, S., Segawa, K., and Nagata, S. (2014). MerTK-mediated Engulfment of Pyrenocytes by central Macrophages in Erythroblastic Islands. Blood 123 (25), 3963-3971. doi:10.1182/blood-2014-01-547976

Ulyanova, T., Phelps, S. R., and Papayannopoulou, T. (2016). The Macrophage Contribution to Stress Erythropoiesis: When Less is Enough. Blood 128 (13), 1756-1765. doi:10.1182/blood-2016-05-714527 
Wang, J., Hayashi, Y., Yokota, A., Xu, Z., Zhang, Y., Huang, R., et al. (2018). Expansion of EPOR-Negative Macrophages besides Erythroblasts by Elevated EPOR Signaling in Erythrocytosis Mouse Models. Haematologica 103 (1), 40-50. doi:10.3324/haematol.2017.172775

Waye, J. S., and Eng, B. (2015). Krüppel-like Factor 1: Hematologic Phenotypes Associated withKLF1gene Mutations. Int. J. Lab. Hem. 37 (Suppl. 1), 78-84. doi:10.1111/ijlh.12356

Wei, Q., Boulais, P. E., Zhang, D., Pinho, S., Tanaka, M., and Frenette, P. S. (2019). Maea Expressed by Macrophages, but Not Erythroblasts, Maintains Postnatal Murine Bone Marrow Erythroblastic Islands. Blood 133 (11), 1222-1232. doi:10.1182/blood-2018-11-888180

Xiang, J., Wu, D.-C., Chen, Y., and Paulson, R. F. (2015). In Vitro Culture of Stress Erythroid Progenitors Identifies Distinct Progenitor Populations and Analogous Human Progenitors. Blood 125 (11), 1803-1812. doi:10.1182/blood-2014-07-591453

Xu, L., Zhu, D., Zhang, Y., Liang, G., Liang, M., Wei, X., et al. (2021). Compound Heterozygosity for KLF1 Mutations Causing Hemolytic Anemia in Children: A Case Report and Literature Review. Front. Genet. 12, 691461. doi:10.3389/ fgene. 2021.691461

Xue, L., Galdass, M., Gnanapragasam, M. N., Manwani, D., and Bieker, J. J. (2014). Extrinsic and Intrinsic Control by EKLF (KLF1) Within a Specialized Erythroid Niche. Development 141 (11), 2245-2254. doi:10.1242/dev.103960

Yeo, J. H., Colonne, C. K., Tasneem, N., Cosgriff, M. P., and Fraser, S. T. (2019a). The Iron Islands: Erythroblastic Islands and Iron Metabolism. Biochim. Biophys. Acta Gen. Subj. 1863 (2), 466-471. doi:10.1016/j.bbagen.2018.10.019

Yeo, J. H., Lam, Y. W., and Fraser, S. T. (2019b). Cellular Dynamics of Mammalian Red Blood Cell Production in the Erythroblastic Island Niche. Biophys. Rev. 11, 873-894. doi:10.1007/s12551-019-00579-2

Yeo, J. H., McAllan, B. M., and Fraser, S. T. (2016). Scanning Electron Microscopy Reveals Two Distinct Classes of Erythroblastic Island Isolated from Adult
Mammalian Bone Marrow. Microsc. Microanal. 22 (2), 368-378. doi:10.1017/ S1431927616000155

Yien, Y. Y., and Bieker, J. J. (2013). EKLF/KLF1, a Tissue-Restricted Integrator of Transcriptional Control, Chromatin Remodeling, and Lineage Determination. Mol. Cell Biol. 33 (1), 4-13. doi:10.1128/mcb.01058-12

Zhang, H., Wang, S., Liu, D., Gao, C., Han, Y., Guo, X., et al. (2021). EpoRtdTomato-Cre Mice Enable Identification of EpoR Expression in Subsets of Tissue Macrophages and Hematopoietic Cells. Blood 2021011410. doi:10.1182/ blood.2021011410

Zhao, L., Lei, W., Deng, C., Wu, Z., Sun, M., Jin, Z., et al. (2021). The Roles of Liver $\mathrm{X}$ Receptor $\alpha$ in Inflammation and Inflammation-Associated Diseases. J. Cell Physiol. 236 (7), 4807-4828. doi:10.1002/jcp.30204

Conflict of Interest: The authors declare that the research was conducted in the absence of any commercial or financial relationships that could be construed as a potential conflict of interest.

Publisher's Note: All claims expressed in this article are solely those of the authors and do not necessarily represent those of their affiliated organizations, or those of the publisher, the editors and the reviewers. Any product that may be evaluated in this article, or claim that may be made by its manufacturer, is not guaranteed or endorsed by the publisher.

Copyright (C) 2021 Mukherjee and Bieker. This is an open-access article distributed under the terms of the Creative Commons Attribution License (CC BY). The use, distribution or reproduction in other forums is permitted, provided the original author(s) and the copyright owner(s) are credited and that the original publication in this journal is cited, in accordance with accepted academic practice. No use, distribution or reproduction is permitted which does not comply with these terms. 